\section{Fatores associados à violência obstétrica na assistência ao parto vaginal em uma maternidade de alta complexidade em Recife, Pernambuco}

\section{Factors associated with obstetric abuse in vaginal birth care at a high-complexity maternity unit in Recife, Pernambuco}

Priscyla de Oliveira Nascimento Andrade 1 Jéssica Queiroz Pereira da Silva 2 Cinthia Martins Menino Diniz 3 Maria de Fátima Costa Caminha 4

\author{
1 Universidade Federal de Pernambuco. Av. Prof. Moraes Rego, 1235 \\ Cidade Universitária. Recife, PE, Brasil. CEP: 50670-901. \\ E-mail: pricila_nas@yahoo.com.br \\ 2-4 Instituto de Medicina Integral Prof. Fernando Figueira. Recife, PE, \\ Brasil.
}

\section{Resumo}

Objetivos: analisar os fatores associados à violência obstétrica de acordo com as práticas não recomendadas na assistência ao parto vaginal em uma maternidade escola e de referência da Cidade do Recife.

Métodos: estudo transversal, prospectivo, com 603 puérperas, realizado entre agosto a dezembro de 2014. Os dados sociodemográficos, clínicos e de acesso à assistência foram obtidos através dos prontuários e de entrevistas com as pacientes. A prevalência da violência obstétrica foi baseada nas recomendações da Organização Mundial da Saúde sobre as práticas recomendadas para a assistência ao parto vaginal. Para análise dos fatores associados utilizou-se a regressão multivariada de Poisson, considerou-se $p<5 \%$. Os dados foram analisados no Stata 12.1 SE.

Resultados: a prevalência da violência obstétrica foi de $86,57 \%$. As práticas prejudiciais mais frequentes foram os esforços de puxo (65\%), a administração de ocitocina (41\%) e o uso rotineiro da posição supina/litotomia (39\%). Apenas as variáveis não possuir ensino médio completo $(p=0,022)$ e ter sido assistido por um profissional médico $(p<0,001)$ apresentaram associação significante com a violência obstétrica.

Conclusões: o grande número de intervenções obstétricas utilizadas consiste em um ato de violência obstétrica e demonstram que apesar do incentivo do Ministério da Saúde para uma assistência humanizada os resultados ainda estão longe do recomendado.

Palavras-chave Tocologia, Violência, Parto humanizado 


\section{Introdução}

Há quase duas décadas a Organização Mundial da Saúde (OMS) compilou informações sobre a assistência ao parto normal, expondo as condutas obstétricas recomendadas e que devem ser mantidas, além daquelas que necessitam ser realizadas com cautela devido à falta de comprovação de seus benefícios; as danosas ou prejudiciais, que devem ser abolidas; e as práticas inadequadas. ${ }^{1}$ Baseado nesse documento, em 2000, o Ministério da Saúde (MS) lança o Programa de Humanização do Pré-natal e Nascimento a fim de garantir as recomendações e direitos da mulher no ciclo gravídico-puerperal. ${ }^{2}$

Dentro deste contexto, surge o conceito de violência obstétrica, a qual é expressa principalmente pela negligência na assistência, discriminação social, violência verbal, física e psicológica, sendo também considerado ato de violência obstétrica, o uso inadequado de tecnologias e a adoção de procedimentos durante o ciclo gravídico-puerperal sem o consentimento explícito e informado da gestante/parturiente, ferindo os princípios dos direitos individuais da mulher. Esses atos de violência podem ser cometidos por pessoas íntimas, estranhas, profissionais ou até mesmo por instituições, podem contribuir para complicações ou efeitos indesejáveis ao binômio mãe-filho.1,3-8

Pesquisas em diversos estados brasileiros evidenciaram o uso arbitrário de autoridade pelos profissionais de saúde em relação ao corpo e à sexualidade das mulheres durante o parto e pós-parto.3,5 No Brasil, uma entre quatro mulheres sofre violência durante o parto, ${ }^{9}$ sendo as condutas desrespeitosas e grosseiras as reclamações mais frequentes entre as puérperas. $6,10,11$

Muitas vezes, a relação entre profissionais de saúde e pacientes de camadas socioeconômicas desfavorecidas é marcada pela desconfiança, desrespeito, conflito e maus tratos, sendo estes também apontados como um fator importante no uso das intervenções desnecessárias, principalmente no que tange a episiotomia em mulheres negra, pobres, nordestinas e estrangeiras. 3,12

De acordo com as recomendações da $\mathrm{OMS}^{1} \mathrm{o}$ parto deve ter início de forma espontânea, não induzida, devendo a parturiente possuir a liberdade de se movimentar a qualquer momento e o direito de receber suporte contínuo durante a parturição, tais como monitoramento cardíacos fetais, alimentação, adoção de posições não supinas, respeito a privacidade, o uso do partograma e presença de acompanhantes, além de evitar intervenções rotineiras. Todo cuidado deve ser individualizado e não deve ocorrer separação de mãe e bebê imediatamente após o parto. 13

Muitos avanços na adesão das boas práticas já foram conseguidos no Brasil, no entanto, o atual modelo de atendimento ao parto, marcado pela medicalização da assistência, transforma as intervenções obstétricas, que deveriam ser somente utilizadas com indicações precisas, em condutas rotineiras e desnecessárias, de acordo com as evidências científicas. ${ }^{14}$

Estudo nacional desenvolvido com 23.894 gestantes indicou que a frequência das intervenções obstétricas foi maior entre aquelas com risco considerado habitual, ou seja, mulheres com risco reduzido de complicações durante a gestação e o parto, demonstrando que as intervenções foram, na maioria das vezes, desnecessárias e realizadas de forma rotineira sem levar a consideração as características clínicas, nem as evidências científicas. 15

A persistente utilização de práticas não recomendadas pelas evidências científicas, como o uso abusivo de ocitocina, imobilização no leito e posição litotômica no parto, pode levar à compressão de grandes vasos e prolongamento do trabalho de parto (TP) e do período expulsivo e, consequentemente, repercutir negativamente sobre os resultados perinatais. 16

O controle da violência obstétrica na assistência ao parto vaginal consiste em um desafio, tendo em vista a sua invisibilidade e não reconhecimento como violação dos direitos humanos. 15 Ampliar os conhecimentos sobre a assistência prestada às mulheres em TP e parto, torna-se uma ferramenta valiosa para a avaliação do processo de atenção no atendimento obstétrico. Nesse sentido, este estudo objetiva analisar os fatores associados à violência obstétrica devido às práticas não recomendadas na assistência ao parto vaginal em uma maternidade de alta complexidade em Recife, Pernambuco.

\section{Métodos}

Estudo transversal, prospectivo, realizado no Instituto de Medicina Integral Professor Fenando Figueira, um hospital escola de alta complexidade e de referência do MS para a assistência maternoinfantil em Recife, Pernambuco, que atende gestantes de baixo, médio e alto risco. A unidade hospitalar foi a primeira a receber a distinção de "Amigo da Criança" no Brasil17 e realiza cerca de 260 partos por mês, por médicos ou enfermeiros, de acordo com o risco da gestação.

A coleta de dados realizou-se entre agosto e dezembro de 2014 por meio da aplicação de um 
questionário elaborado com base nas recomendações da OMS a respeito das práticas na assistência ao parto vaginal. ${ }^{1}$ Foram realizadas entrevistas com as puérperas durante a internação hospitalar e extraídos dados do prontuário da mulher e do recém-nato.

Foram consideradas como elegíveis todas as mulheres que tiveram seus filhos por via vaginal no período da coleta. Os critérios de exclusão foram: presença de condição clínica desfavorável, ou seja, alguma complicação grave que resultou em internação na UTI; ausência de capacidade cognitiva e idade menor do que 18 anos.

O tamanho da amostra foi calculado no módulo STATCALC do EPI-Info 3.5.2, adotando-se um nível de confiança de $95 \%$ e um erro de $4 \%$, admitindo-se prevalência de $25 \%$ de frequência da violência obstétrica, ${ }^{9}$ resultando em uma estimativa amostral de 450 puérperas.

As variáveis de exposição foram: variáveis sociodemográficas: idade (18 a 35 ou $>36$ anos), raça/cor autorrelatada (branca, amarela, parda negra), instrução (ensino médio incompleto ou completo), estado civil (solteira, casada, divorciada, união estável), procedência (região metropolitana do Recife, outras cidades, outros estados), ocupação (alguma atividade remunerada).

Variáveis clínicas: gravidez de risco (história de diabetes ou hipertensão arterial gestacional ou prégestacional, cardiopatias ou outras doenças crônicas, obesas (IMC > 30), HIV positivas, com idade gestacional entre $<37$ ou $>41$ semanas ao nascer, gravidez múltiplas, apresentação pélvica ou má formação fetal; essa variável foi identificada no prontuário através do diagnóstico de internamento da paciente), idade gestacional no momento do parto $(<37 \mathrm{sem}$, 37-41 sem, $>41$ sem), complicações no trabalho de parto/parto (identificadas com base na CID 10), Apgar no $1^{\circ}$ e no $5^{\circ}$ minuto $(<7 \mathrm{e} \geq 7)$.

Variáveis de acesso à assistência: realização do pré-natal ( $\operatorname{sim}$ ou não) e número de consultas ( $<6$ ou $\geq 6$ ), tipo de parto vaginal (não instrumentalizado/ instrumentalizado), profissional que atendeu ao parto (médico, enfermeiro ou outros).

Considerou-se como desfecho, a violência obstétrica, definida como a utilização de pelo menos uma das práticas consideradas claramente desnecessárias prejudiciais, ineficazes e/ou sem evidências científicas de acordo com a OMS1: uso da posição supina ou litotômica no momento do parto, infusão venosa de rotina, exame retal, administração de ocitocina sem indicação precisa, incentivo ao puxo prolongado, amniotomia precoce, manobra de Kristeller, toque vaginais repetitivos, restrição hídrica e alimentar, episiotomia e clampea- mento precoce do cordão. ${ }^{1}$

Informações sobre alimentação durante o TP, o uso de métodos não farmacológicos para alívio da dor, deambulação, uso de cateter venoso, litotomia, toque vaginais, incentivo ao puxo e manobra de Kristeller, foram coletadas durante as entrevistas, enquanto que o uso de partograma, ocitocina para aceleração do trabalho de parto, aminiotomia e tipo de parto, foram dados extraídos dos prontuários médicos.

As práticas que foram realizadas devido à justificativa clínica explícita descrita no prontuário, como, o não preenchimento do partograma e o não incentivo a deambulação em pacientes internadas em período expulsivo e a restrição de alimentos em pacientes que estavam em uso de sulfato de magnésio, não foram consideradas desnecessárias.

As características da população de estudo foram descritas em frequências absolutas e relativas. A análise dos fatores associados à violência obstétrica foi realizada pela regressão multivariada de Poisson, estimando-se as razões de prevalência (RP) brutas, ajustadas e os respectivos intervalos de confiança de 95\%. Foram incluídas no modelo de regressão múltipla, as variáveis que na análise univariada apresentavam-se associadas ao desfecho (violência obstétrica) a um nível de significância de $p<20 \%$. Para compor o modelo final o nível de significância adotado foi $<5 \%$. As análises foram realizadas no software Stata 12.1 SE.

O projeto de estudo foi aprovado pelo Comitê de Ética em Pesquisa em Seres Humanos do Instituto de Medicina Integral Prof. Fernando Figueira sob o protocolo $n^{\circ} 4259-14$.

\section{Resultados}

No período estudado ocorreram 952 partos por via vaginal, dos quais 159 foram excluídos (pacientes menores de idade), obtendo-se uma amostra de 793 partos elegíveis. As perdas ocorreram devido à alta hospitalar fora do horário regular do serviço $(n=158$ pacientes) e recusas para participar do estudo $(n=32)$. Desta forma, a amostra final foi constituída de 603 puérperas.

Observamos que $89 \%$ das puérperas tinham idade entre 18 e 35 anos, $71 \%$ tinham companheiro e $79 \%$ moravam na região metropolitana do Recife. Mais da metade tinha pelo menos ensino médio completo e a maioria (68\%) não tinha emprego. Em relação aos dados obstétricos mais de $90 \%$ das gestantes realizaram consultas de pré-natal e o parto vaginal não instrumentalizado foi predominante (98\%). Observou-se que $71 \%$ dos partos foram assis- 
tidos por médicos e $28 \%$ por enfermeiros.

Pouco mais que a metade das puérperas (53\%) teve como diagnóstico de internamento o TP, $33 \%$ foram diagnosticadas com alguma síndrome hipertensiva gestacional e 19\% deram entrada com trabalho de parto prematuro. A maior parte das pacientes $(82 \%)$ apresentou algum tipo de complicação durante o trabalho de parto e/ou parto, sendo a mais frequente a laceração do períneo (78\%), seguida pelas contrações inadequadas (49\%) (Tabela 1).

Aproximadamente $87 \%$ das pacientes sofreram algum tipo de violência durante o TP e parto, considerando o uso de intervenções desnecessárias. Mais de $65 \%$ das mulheres referiram o incentivo aos puxos voluntários, a incidência da posição supina e de litotomia foi de $27 \%$ e $12 \%$, respectivamente, enquanto o uso de ocitocina e de aminiotomia foi de $41 \%$ e $31 \%$, respectivamente. O clampeamento precoce do cordão umbilical ainda foi realizado em $30 \%$ das pacientes.

Quanto às boas práticas, aplicadas em quase todas as pacientes, as mais utilizadas foram: direito a escolha do acompanhante (95\%), apoio emocional
(93\%) e respeito a privacidade $(89 \%)$, sendo o partograma a prática menos utilizada (28\%). Os métodos não invasivos para alívio da dor foram utilizados em $50 \%$ das parturientes, destes o mais utilizado foi a bola suíça (76\%). Outras informações a respeito das práticas prejudiciais e das úteis encontram-se detalhadas na Tabela 2.

A Tabela 3 apresenta as características sociodemográficas e obstétricas das mulheres e os resultados das análises uni e multivariadas dos fatores associados à violência obstétrica de acordo com as práticas desnecessárias que foram utilizadas.

$\mathrm{Na}$ análise univariada, as variáveis ensino médio completo, procedência, gravidez de risco, pré-natal, tipo de parto, idade gestacional e profissional que assistiu ao parto mostraram associação estatística com a violência obstétrica a um nível de significância de $p<0,20$, credenciando-se assim para a etapa da análise multivariada. Após os ajustes, não possuir o ensino médio completo $(p=0,022)$ e ter sido assistida durante o parto por profissional médico $(p<0,001)$, apresentaram associação significante com a violência obstétrica $(p<0,05)$.

Tabela 1

Diagnóstico de internamento e complicações do trabalho de parto e parto mais frequentes em gestantes assistidas em maternidade de alta complexidade. Recife, Pernambuco, 2015.

\begin{tabular}{|c|c|c|}
\hline Variáveis/Categorias & $\mathrm{N}=603^{*}$ & $\%$ \\
\hline \multicolumn{3}{|l|}{ Diagnóstico de internamento } \\
\hline Trabalho de parto & 319 & 53,0 \\
\hline Síndromes hipertensivas & 197 & 33,0 \\
\hline Trabalho de parto prematuro & 116 & 19,0 \\
\hline Outros & 136 & 23,0 \\
\hline Complicações & 496 & 82,0 \\
\hline Laceração & 389 & 78,0 \\
\hline $4^{\circ}$ grau & 1 & 0,3 \\
\hline Contrações inadequadas & 245 & 49,0 \\
\hline Trabalho de parto prematuro & 115 & 23,0 \\
\hline Outras & 35 & 7,0 \\
\hline
\end{tabular}

*A amostra variou devido possibilidade das pacientes apresentarem mais de um diagnóstico ou complicação. 
Caracterização das práticas prejudiciais e das boas práticas e intervenções durante o trabalho de parto e parto em maternidade de alta complexidade. Recife, Pernambuco, 2015.

\begin{tabular}{|c|c|c|}
\hline Variáveis/Categorias & $\mathrm{N}=603^{*}$ & $\%$ \\
\hline Práticas claramente prejudiciais, ineficazes e/ou sem evidências científicas & 522 & 86,57 \\
\hline Infusão venosa de rotina no trabalho de parto/parto & 0 & - \\
\hline Uso da posição supina & 162 & 27,0 \\
\hline Uso da posição litotômica & 72 & 12,0 \\
\hline Exame retal & 59 & 10,0 \\
\hline Administração de ocitócitos & 245 & 41,0 \\
\hline Incentivo aos puxos voluntários & 390 & 65,0 \\
\hline Amniotomia precoce & 186 & 31,0 \\
\hline Manobra de Kristeller & 52 & 9,0 \\
\hline Manipulação ativa do feto/ Toques vaginais repetidos & 112 & 19,0 \\
\hline Restrição hídrica e alimentar no trabalho de parto & 54 & 11,0 \\
\hline Uso da epsiotomia & 13 & 2,0 \\
\hline Clampeamento precoce do cordão umbilical & 180 & 30,0 \\
\hline Práticas demonstradamente úteis & 602 & 99,8 \\
\hline Respeito à privacidade & 537 & 89,0 \\
\hline Apoio dos profissionais no trabalho de parto & 558 & 93,0 \\
\hline Liberdade de posição/movimentação no trabalho de parto & 461 & 82,0 \\
\hline Estímulos às posições não supinas no trabalho de parto & 373 & 67,0 \\
\hline Recebeu informações sobre o trabalho de parto /parto & 457 & 76,0 \\
\hline Ingestão de líquidos durante o trabalho de parto & 411 & 80,0 \\
\hline Respeito à escolha do acompanhante & 574 & 95,0 \\
\hline Utilização do partograma & 168 & 28,0 \\
\hline Contato precoce pele-a-pele com o bebê na $1^{\mathrm{a}}$ horas após o parto & 509 & 85,0 \\
\hline Métodos não invasivos para alívio da dor & 301 & 50,0 \\
\hline Bola & 229 & 76,0 \\
\hline Massagem & 85 & 28,0 \\
\hline Banho & 125 & 42,0 \\
\hline Cavalinho & 22 & 7,0 \\
\hline Outros & 68 & 23,0 \\
\hline
\end{tabular}

*A amostra variou devido possibilidade de várias práticas terem sido realizadas em uma paciente. 
Tabela 3

Distribuição de frequência, razão de prevalência bruta e ajustada da associação da violência obstétrica de acordo com características sociodemográficas, obstétricas maternas e clínicas do recém-nascido em maternidade de alta complexidade. Recife, Pernambuco, 2015.

\begin{tabular}{|c|c|c|c|c|c|c|c|c|}
\hline \multirow[t]{2}{*}{ Variáveis } & \multicolumn{2}{|c|}{$\begin{array}{l}\text { Amostra } \\
\left(\mathrm{N}=603^{*}\right)\end{array}$} & \multicolumn{2}{|c|}{$\begin{array}{l}\text { Violência } \\
\text { obstétrica }\end{array}$} & \multirow[t]{2}{*}{$\begin{array}{l}\text { RP } \text { bruta } \\
\text { (IC95\%) }\end{array}$} & \multirow[t]{2}{*}{$p^{* *}$} & \multirow[t]{2}{*}{$\begin{array}{r}\mathrm{RP}_{\text {ajustada }} \\
\text { (IC95\%) }\end{array}$} & \multirow[t]{2}{*}{$p^{* *}$} \\
\hline & $\mathrm{n}$ & $\%$ & $\mathrm{n}$ & $\%$ & & & & \\
\hline Idade (anos) & & & & & & 0,6895 & & \\
\hline 18 a 35 & 536 & 89,0 & 463 & 86,3 & 1,0 & & & \\
\hline 36 e mais & 67 & 11,0 & 59 & 88,0 & $1,02(0,93-1,12)$ & & & \\
\hline Raça & & & & & & 0,4909 & & \\
\hline Branca & 134 & 22,0 & 112 & 83,5 & 1,0 & & & \\
\hline Negra & 94 & 16,0 & 80 & 85,1 & $1,02(0,91-1,14)$ & & & \\
\hline Parda & 350 & 58,0 & 307 & 87,7 & $1,05(0,96-1,14)$ & & & \\
\hline Amarela & 25 & 4,0 & 23 & 92,0 & $1,10(0,96-1,26)$ & & & \\
\hline Ensino médio completo & & & & & & 0,0054 & & 0,022 \\
\hline Sim & 316 & 52,0 & 262 & 82,9 & 1,0 & & 1,0 & \\
\hline Não & 287 & 48,0 & 260 & 90,5 & $1,09(1,03-1,16)$ & & $1,06(1,01-1,12)$ & \\
\hline Companheiro & & & & & & 0,7128 & & \\
\hline Sim & 429 & 71,0 & 370 & 86,2 & 1,0 & & & \\
\hline Não & 174 & 29,0 & 152 & 87,3 & $1,01(0,95-1,08)$ & & & \\
\hline Procedência & & & & & & $<0,001$ & & 0,3685 \\
\hline RMR & 477 & 79,0 & 401 & 84,07 & 1,0 & & 1,0 & \\
\hline Interiores & 118 & 20,0 & 113 & 95,7 & $1,14(1,08-1,20)$ & & $1,03(0,99-1,08)$ & \\
\hline Outros Estados & 8 & 1,0 & 8 & 100,0 & $1,19(1,14-1,24)$ & & $1,01(0,94-1,09)$ & \\
\hline Trabalho & & & & & & 0,8119 & & \\
\hline Sim & 194 & 32,0 & 167 & 86,0 & 1,0 & & & \\
\hline Não & 409 & 68,0 & 355 & 86,8 & $1,01(0,94-1,08)$ & & & \\
\hline Gravidez de risco & & & & & & $<0,001$ & & 0,113 \\
\hline Sim & 364 & 60,0 & 354 & 97,2 & $1,38(1,27-1,51)$ & & $0,97(0,93-1,01)$ & \\
\hline Não & 239 & 40,0 & 168 & 70,2 & 1,0 & & 1,0 & \\
\hline Fez pré-natal & & & & & & $<0,001$ & & 0,066 \\
\hline Sim & 581 & 96,0 & 500 & 86,0 & 1,0 & & 1,0 & \\
\hline Não & 22 & 4,0 & 22 & 100,0 & $1,16(1,12-1,20)$ & & $1,04(0,99-1,09)$ & \\
\hline $\mathrm{N}^{\circ}$ de consultas* & & & & & & 0,6199 & & \\
\hline$<6$ & 215 & 37,0 & 187 & 86,9 & $1,02(0,95-1,09)$ & & & \\
\hline$\geq 6$ & 366 & 63,0 & 313 & 85,5 & 1,0 & & & \\
\hline Tipo de parto & & & & & & $<0,001$ & & 0,312 \\
\hline Vaginal & 591 & 98,0 & 510 & 86,2 & 1,0 & & 1,0 & \\
\hline Vaginal Instrument. $* * *$ & 12 & 2,0 & 12 & 100,0 & $1,16(1,12-1,20)$ & & $1,02(0,98-1,08)$ & \\
\hline Idade gestacional* & & & & & & $<0,001$ & & 0,6351 \\
\hline$<37$ & 112 & 19,0 & 110 & 98,2 & $1,17(1,11-1,23)$ & & $0,98(0,94-1,02)$ & \\
\hline 37 a 41 & 444 & 74,0 & 372 & 83,7 & 1,0 & & 1,0 & \\
\hline$>41$ & 43 & 7,0 & 36 & 83,7 & $1,00(0,87-1,14)$ & & $0,97(0,87-1,08)$ & \\
\hline Profissional & & & & & & $<0,001$ & & $<0,001$ \\
\hline Médico & 427 & 71,0 & 417 & 97,6 & $1,65(1,46-1,87)$ & & $1,56(1,38-1,76)$ & \\
\hline Enfermeiro & 171 & 28,0 & 101 & 59,0 & 1,0 & & 1,0 & \\
\hline Outros & 5 & 1,0 & 4 & 80,0 & $1,35(0,86-2,13)$ & & $1,57(1,38-1,78)$ & \\
\hline
\end{tabular}

*A amostra variou devido à ausência de informação no prontuário; **Poisson; RMR= região metropolitana do Recife; *** vaginal instrumentalizado. 


\section{Discussão}

O modelo de assistência ao TP e parto no Brasil é conhecido pelo intervencionismo e os resultados deste estudo corroboram essa prática, entretanto, as boas práticas foram aplicadas em $99 \%$ das parturientes, o que mostra um grande avanço na humanização da assistência ao parto. Em contrapartida, algum tipo de práticas consideradas prejudiciais ou desnecessárias ainda foram utilizadas em $89 \%$ das pacientes, sendo o incentivo aos puxos e a administração de ocitocina os mais frequentes, corroborando com estudo de abrangência nacional, que constatou prevalência elevada de intervenções obstétricas. 15

Esse fato sugere que, mesmo após duas décadas das recomendações da OMS, ${ }^{1}$ para a assistência ao parto vaginal, a adesão dos profissionais tem se mostrado insuficiente para reverter o modelo intervencionista da atenção obstétrica, o que pode estar associado ao fato de que a medicina, por longos anos, foi baseada por experiências profissionais, representando comportamentos condicionados por razões culturais, políticas, econômicas e crenças religiosas, 4 sendo resistentes às práticas baseadas em evidências.

A taxa de episiotomia encontrada no presente estudo foi em torno de $2 \%$, percentual abaixo dos $10 \%$ recomendado pela OMS. 1 A episiotomia tornou-se uma rotina na assistência ao parto com a finalidade de reduzir danos, mas estudos controlados demonstram que este procedimento aumenta o risco de laceração perineal grave, de infecção e hemorragias, além de complicações como incontinência urinária e fecal.18,19 Portanto, as novas diretrizes desestimulam o seu uso rotineiro. 20

Durante o parto, a posição de litotomia e supina, juntas, alcançaram apenas $39 \%$ entre as parturientes, o que mostra que a maioria destas pariram em posições não supinas, o que possivelmente demostra esforços dos profissionais de saúde em modificar o atual cenário da assistência obstétrica. Em contraponto, estudo conduzido por Leal et al. ${ }^{15} \mathrm{e}$ Carvalho et al.,21 no Rio de Janeiro, indicou que pelo menos $90 \%$ e $100 \%$, respectivamente, das parturientes de risco habitual pariram na posição litotômica, apesar dos vários benefícios das posições verticalizadas para a mulher e o feto.

As revisões sistemáticas realizadas por Gupta et al. ${ }^{22}$ e Lawrence et al., ${ }^{23}$ recomendam a adoção de posições verticalizadas, pois podem reduzir o período do TP e não estão associadas com o aumento da intervenção ou efeitos negativos sobre o bemestar das mães e bebês.

Nesse estudo, verificou-se que a infusão de ocitocina e a realização da amniotomia foram condutas técnicas frequentemente utilizadas com o objetivo de acelerar o trabalho de parto, tendo esses procedimentos sido adotados em $41 \%$ e $31 \%$ dos partos, respectivamente. Esses resultados estão de acordo com os dados de um estudo de abrangência nacional, 15 que encontrou uma prevalência de 38,2\% e $40,7 \%$ e outro realizado na cidade de São Paulo, 24 com prevalências $31 \%$ e $53,4 \%$, ambos os serviços do Sistema Único de Saúde (SUS).

Revisões sistemáticas realizadas por Wei et al., ${ }^{25}$ apontam uma redução do número de cesarianas quando o manejo ativo do TP é implementado, entretanto, reconhece-se que este não deve ser recomendado de rotina na prática obstétrica. A elevada frequência de uso de ocitocina pode ter relação com o grande número de lacerações do períneo, a complicação mais frequente na população em estudo.

Quanto ao uso das práticas comprovadamente úteis, como a alimentação TP, movimentação durante o primeiro estágio de $\mathrm{TP}$, respeito à escolha do acompanhante e respeito à privacidade, apresentou frequência variada e, de um modo geral, percentuais que alcançavam mais de $70 \%$ dos partos. Esses dados divergem de outro estudo 15 encontrado, no qual se observou que o uso das boas práticas não alcançaram $50 \%$ das gestantes. Constatamos que $80 \%$ das parturientes ingeriram algum tipo de líquido e que apenas $11 \%$ das pacientes tiveram restrição hídrica e alimentar, não existindo evidências que apoiem a restrição de líquidos e alimentos durante o TP para as mulheres de risco habitual.26

Esse aumento na adoção de boas práticas é, provavelmente, consequente às ações do MS de qualificação dos profissionais de saúde e adequação das salas de parto para incentivar a promoção do parto normal e humanizado, através do Programa de Humanização do Pré-natal e Nascimento. ${ }^{21}$

Dentre as variáveis estudas, observou-se associação significante com a violência obstétrica mulheres que não possuíam ensino médio completo e aquelas assistidas por profissional médico. Os achados são semelhante aos encontrados nos estudos de Leal et al. ${ }^{15}$ e Janssen et al., 27 nos quais mulheres socioeconomicamente desfavorecidas apresentaram maior utilização de procedimentos dolorosos, como a aceleração do trabalho de parto e baixo uso de analgesia obstétrica, e naquelas com o menor nível de escolaridade, onde foi comum uso de ocitocina, amniotomia e episiotomia, e menos frequente o uso de analgesia.

O maior grau de instrução pode ter relação com um número menor de intervenções, pois essas pacientes procuram ter mais acesso à informação 
científica o que permite o empoderamento destas frente aos cuidados baseado em evidências. ${ }^{15}$ Assim os profissionais são mais questionados pelas pacientes, desencorajando a realização de alguns procedimentos contra indicados ou potencialmente danosos.

Estudo desenvolvido na Colúmbia Britânica evidenciou que as boas práticas são mais utilizadas pelos enfermeiros obstetras ou obstetrizes quando comparados com profissionais médicos. ${ }^{27} \mathrm{~A}$ inserção do enfermeiro obstetra na assistência ao parto sucedeu a inserção médica, logo, estes profissionais tiveram uma formação voltada para a atual problemática e com base na assistência humanizada. Diferente da formação médica, com características intervencionista, cujo objetivo era reduzir os altos índices da mortalidade materna.

Embora a frequência das boas práticas serem superiores as intervenções desnecessárias, a persistência destas consiste em um ato de violência obstétrica e evidencia que progressos ainda são necessários. Apesar do grande número de intervenções obstétricas utilizadas que caracterizaram a violência, observamos frequências inferiores,

\section{Referências}

1. OMS (Organização Mundial da Saúde). Tecnologia apropriada para partos e nascimentos. Recomendações da Organização Mundial de Saúde. Maternidade Segura. Assistência ao parto normal: um guia prático. Genebra; 1996.

2. Brasil. Ministério da Saúde. Programa de Humanização no Pré-Natal e Nascimento (PHPN). Brasília, DF; 2000.

3. Aguiar JM, Oliveira AFPL. Violência institucional em maternidades públicas sob a ótica das usuárias. InterfaceComunic., Saude, Educ. 201115 (36): 79-91.

4. Muniz BMV, Barbosa RM. Problematizando o atendimento ao parto: cuidado ou violência? Memorias Convención Internacional de Salud Pública. [periódico online]. 2012. [acesso em 1 Set 2013]. Disponível em: http://www.defensoria.sp.gov.br/dpesp/Repositorio/41/Documentos/artigo\% 20parto.pdf

5. Milfont PMS, Silva VM, Chaves DBR, Beltrão BA. Estudo exploratório sobre a implementação de diretrizes para um parto seguro e satisfação das mulheres. Online Braz J Nurs. 2011 [acesso em 10 Set 2014]; 10 (3). Disponível em: http://www.objnursing.uff.br/index.php/nursing/article/vie w/3493.

6. Pereira WR. O Poder, a violência e a dominação simbólicos nos serviços públicos de saúde que atendem mulheres em situação de gestação parto e puepério. Texto Contexto Enferm. 2004; 13 (3): 391-400.

7. Aguiar JM, d'Oliveira AFPL, Schraiber LB. Violência institucional, autoridade médica e poder nas maternidades sob a ótica dos profissionais de saúde. Cad Saúde Pública. 2013; quando comparadas com outro estudo, 15 o que demostra resultados positivos do incentivo do MS para uma assistência humanizada, apesar de esta longe do recomendado.

Apenas um estudo 15 que considerou as práticas prejudiciais/intervenções como uma forma de violência obstétrica, foi encontrado. Essas são vistas como procedimentos normais e até comum em muitas maternidades. Dessa forma é necessário que mais estudos abordem essa temática. É imprescindivel que os profissionais tenham mais rigor nas anotações das intervenções realizadas nos prontuários.

Sugere-se que os resultados e suas análises possam representar o pretexto para uma discussão interna com os "staffs" técnicos e administrativos dos hospitais/maternidades que, desafiados pelo atendimento de casos de baixo, médio e elevado risco obstétrico, possam buscar estratégias alternativas ou complementares para melhorar seu desempenho, no que concerne a difusão das boas práticas obstétricas, prestando uma assistência livre da violência obstétrica.

8. Minayo MCS, Souza ER. Violência sob o olhar da saúde: infrapolítica da contemporaneidade brasileira. Cad. Saúde Pública. 2006; 20 (4): 23-47.

9. Venturi G, Godinho T. Mulheres brasileiras e gênero nos espaços público e privado: uma década de mudanças na opinião pública. São Paulo: Editora Fundação Perseu Abramo/SESC-SP; 2013.

10. Domingues RMSM, Santos EM, Leal MC. Aspectos das satisfações das mulheres com assistência ao parto: contribuição para um debate. Cad Saúde Pública. 2004; 20 (1): 52-62.

11. Teixeira NZF, Pereira WR. Parto hospitalar: experiências das mulheres na periferia de Cuiabá. Rev Bras Enf. 2006. 59 (6): 740-4.

12. Hotimsky SN, Rattner D, Venancio SI, Bógus CM, Miranda MM. O parto como eu vejo... ou como eu o desejo?: expectativas de gestantes, usuárias do SUS, acerca do parto e da assistência obstétrica. Cad Saúde Pública. 2002. 18 (5): 1303-11.

13. Rocha JA, Novaes PB. Uma reflexão após 23 anos das recomendações da Organização Mundial da Saúde para parto normal. Femina. [online] 2010; 38 (3) [acesso em 10 nov 2015]. Disponível em: http://files.bvs.br/ upload/S/0100-7254/2010/v38n3/a002.pdf

14. Diniz SG. "O corte por cima" e o "corte por baixo": o abuso de cesáreas e episiotomias em São Paulo. Questões de Saúde Reprodutiva I [online]. 2006. [acesso em 28 Ago 
2013]. 1 (1): 80-91. Disponível em: http://www.mulheres. org.br/revistarhm/revista_rhm $1 /$ revista $1 / 80-91$.pdf

15. Leal MC, Pereira APE, Domingues RMSM, Filha MMT, Dias MAB, Pereira MN, Bastos MH, Gama SGN. Intervenções obstétricas durante o trabalho de parto e parto. Cad Saúde Pública. 2014; 30: 17-47.

16. Lansky S, Friche AAL, Silva AAM, Campos D, Bittencourt SDA, Carvalho ML, Frias PG, Cavalcante RS, Cunha AJLA. Pesquisa Nascer no Brasil: perfil da mortalidade neonatal e avaliação da assistência à gestante e ao recémnascido. Cad Saúde Pública. 2014; 30: S192-S207.

17. Belo MNM, Azevedo PTACC, Belo MPM, Serva VMSBD, Batista Filho M, Figueiroa JN, Caminha MFC. Aleitamento materno na primeira hora de vida em um Hospital Amigo da Criança: prevalência, fatores associados e razões para sua não ocorrência. Rev Bras Saúde Matern Infant. 2014; 14 (1): $65-72$.

18. Carvalho CCM, Souza ASR, Moraes Filho OB. Episiotomia seletiva: avanços baseados em evidências. Femina. [online] 2010; 38 (5): 265-70.

19. Raisanen S, Vehvilainen-Julkunen K, Gisler M, Heinonen $\mathrm{S}$. A population-based register study to determine indications for episiotomy in Finland. Int J Gynaecol Obstet. [online]. 2011; 115: 26-30.

20. Carroli G, Mignini L. Episiotomy for vaginal birth. Cochrane Database Syst Rev. [online] 2009; (1): CD000081. [acesso em 1 nov 2015]. Disponível em:http://onlinelibrary.wiley.com/doi/10.1002/14651858.C D 000081.pub2/pdf

21. Carvalho VF,Kerber NPC,Busanello J,Costa, MMG,Gonçalves BG,Quadros VF. Práticas prejudiciais ao parto: relato dos trabalhadores de saúde do sul do Brasil. Rev Rene. [online]. 2010; 11 (Número Especial): 92-9. Disponível em: http://www.revistarene.ufc.br/edicaoespecial/a10v11esp_n4.pdf
22. Gupta JK, Hofmeyr GJ, Shehmar M. Position in the second stage of labour for women without epidural anaesthesia. Cochrane Database Syst Rev. [online] 2012. [acesso em 10 Jan 2015]. 5: CD002006. Disponível em: http://www.ncbi.nlm.nih.gov/pubmed/22592681

23. Lawrence A, Lewis L, Hofmeyr GJ, Dowswell T, Styles C. Maternal positions and mobility during first stage labour (Cochrane Review). In: The Cochrane Library, Issue 1. [online] 2010. [acesso em 9 Jan 2015]. Disponível em: http://www.ncbi.nlm.nih.gov/pubmed/19370591

24. Silva FMB, Paixão TCR, Oliveira SMJV, Leite JS, Riesco MLG, Osava RH.Assistência em um centro de parto segundo as recomendações da Organização Mundial da Saúde. Rev Esc Enferm USP. 2013; 47 (5): 1031-8.

25. Wei S, Wo BL, Xu H, Luo ZC, Roy C, Fraser WD. Early amniotomy and early oxytocin for prevention of, or therapy for, delay in first stage spontaneous labour compared with routine care. Cochrane Database Syst Rev. [online] 2012. [acesso em 23 Dez 2014]. 9: CD006794. Disponível em: http://www.ncbi.nlm.nih.gov/pubmed/19370654

26. Singata M, Tranmer J, Gyte GM. Restricting oral fluid and food intake during labour. Cochrane Database Syst Rev. [online] 2010. [acesso em 20 jan 2015]. (1): CD003930. Disponível em: http://www.ncbi.nlm.nih.gov/pmc/articles/PMC4175539/

27. Janssen PA, Ryan EM, Etches DJ, Klein MC, Reime B. Outcomes of planned hospital birth attended by midwives compared with physicians in British Columbia. Birth. 2007; 34 (2): 140-7.

Recebido em 12 de maio de 2015

Versão final apresentada em 5 de janeiro de 2016

Aprovado em 15 de janeiro de 2016 